\title{
Sarcoidosis in a Chronic Dialysis Patient Diagnosed by Sarcoidosis-related Hypercalcemia with No Common Systemic Clinical Manifestations: A Case Report and Review of the Literature
}

\author{
Yohei Arai ${ }^{1}$, Hiroyuki Tanaka ${ }^{1}$, Suguru Hirasawa ${ }^{1}$, Shota Aki ${ }^{1}$, Naoto Inaba ${ }^{1}$, \\ Makoto Aoyagi ${ }^{1}$, Yukio Tsuura ${ }^{2}$ and Teiichi Tamura ${ }^{1}$
}

\begin{abstract}
Sarcoidosis is a systemic granulomatous disease of unknown origin. We herein report a case of sarcoidosis in a chronic dialysis patient diagnosed by hypercalcemia without any common clinical manifestations. The onset of sarcoidosis in chronic dialysis patients is rare; to the best of our knowledge, only 23 cases have been reported. Evaluation of the 23 previously published cases revealed that a diagnosis of sarcoidosis was often achieved by the presence of sarcoidosis-related hypercalcemia without any common clinical presentations, as in the present case. This characteristic may arise from a specific immune deficiency and the unique physiology of 1,25-dihydroxyvitamin D3, a main cause of sarcoidosis-related hypercalcemia, in chronic dialysis patients. These clinical features may be useful to understand the pathogenesis of sarcoidosis.
\end{abstract}

Key words: sarcoidosis, dialysis, hypercalcemia, gallium scintigraphy

(Intern Med 52: 2639-2644, 2013)

(DOI: 10.2169/internalmedicine.52.1075)

\section{Introduction}

Sarcoidosis is a systemic granulomatous disease of unknown origin. It can affect virtually any organ with diverse manifestations (1). However, most patients present with typical symptoms that are recognizable at the first clinical encounter. Up to $50 \%$ of patients diagnosed with sarcoidosis are asymptomatic, and their condition is often discovered incidentally as a result of bilateral hilar lymphadenopathy (BHL) on chest X-rays. BHL is associated with about $95 \%$ of cases of sarcoidosis at diagnosis $(2,4)$. Approximately $10 \%$ to $20 \%$ of patients have bilateral uveitis with blurred vision and photophobia at diagnosis $(3,4)$, and about $15 \%$ of patients have a skin pathology such as erythema nodosum (EN) at diagnosis (4). Besides these clinical manifestations, as shown in Table 1, relatively few patients are diagnosed by cardiac involvement, neurologic symptoms, and hypercalcemia $(1,4)$. In terms of nephrologic manifestations, renal failure represented by interstitial nephritis is associated with less than $1 \%$ of cases of sarcoidosis (4). On the other hand, the onset of sarcoidosis has been rarely reported in chronic dialysis patients; as far as we know, only 23 cases have been published (5-24). Therefore, nephrologic manifestations of sarcoidosis are considered to be rare, especially in chronic dialysis patients. We herein report a case of sarcoidosis in a chronic dialysis patient diagnosed in the wake of sarcoidosis-related hypercalcemia without the presence of any common systemic clinical manifestations. A review of published cases of sarcoidosis in chronic dialysis patients is also presented, and the pathophysiologic features of these cases are discussed.

\section{Case Report}

An 81-year-old man was admitted in August 2011 because of general fatigue, loss of appetite, insomnia, and hypercalcemia (adjusted calcium, $12.5 \mathrm{mg} / \mathrm{dL}$ ). He had developed chronic renal failure related to diabetic nephropathy, which led to hemodialysis in 2005. By this time, he had in-

${ }^{1}$ Department of Nephrology, Yokosuka Kyosai Hospital, Japan and ${ }^{2}$ Department of Pathology, Yokosuka Kyosai Hospital, Japan Received for publication May 28, 2013; Accepted for publication July 9, 2013

Correspondence to Dr. Yohei Arai, y.a-hotmail@ hotmail.co.jp 
Table 1. Organ Involvement in Sarcoidosis

\begin{tabular}{lr}
\hline Organ & Cases \\
\hline Lung/Thoracic lymph nodes & $95 \%$ \\
Skin & $23 \%$ \\
Exclusive of EN & $15 \%$ \\
EN & $8 \%$ \\
Peripheral lymph nodes & $15 \%$ \\
Eye & $12 \%$ \\
Liver & $11 \%$ \\
Spleen & $7 \%$ \\
Neurologic & $5 \%$ \\
Parotid/Salivary & $4 \%$ \\
Calcium & $4 \%$ \\
Ear, Nose, Throat & $3 \%$ \\
Cardiac & $2 \%$ \\
Renal & $<1 \%$ \\
Bone/Joint & $<1 \%$ \\
\hline
\end{tabular}

Data from references $[1,4]$.

EN: erythema nodosum

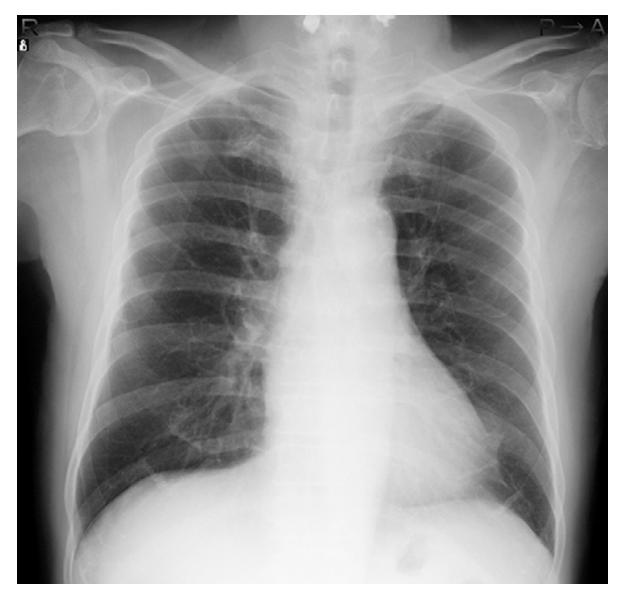

Figure 1. Chest X-ray on admission. There were no typical findings, such as bilateral hilar lymphadenopathy.

dicated no evident findings recalled a combination of tubulointerstitial nephritis and his serum adjusted calcium level had stabilized within the normal range, between about 8.0 and $10.0 \mathrm{mg} / \mathrm{dL}$. After introduction of hemodialysis, his serum calcium, phosphorus, and intact parathyroid hormone (iPTH) levels were well controlled by administration of alfacalcidol and sevelamer until April 2011. The hypercalcemia then returned and progressed gradually despite the withdrawal of alfacalcidol. His medical history included diabetes with diabetic retinopathy, arteriosclerosis obliterans, colon diverticulitis, and cataract. He had taken sevelamer, valsartan, felodipine, pitavastatin, allopurinol, oxatomide, sennoside, and insulin. He had no smoking history. On admission, he was alert and well oriented. His body temperature was 97.7 degrees Fahrenheit, his heart rate was 68 beats per minute, and his blood pressure was $177 / 77 \mathrm{mmHg}$. Physical examination revealed no notable findings. There were no rales, rashes, or superficial lymphadenopathy. A tuberculin

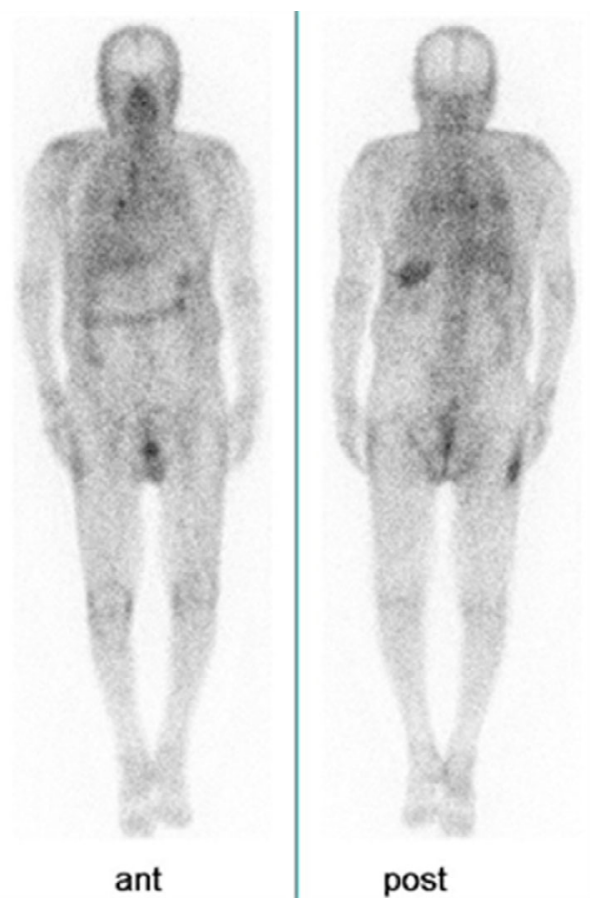

Figure 2. Total-body gallium scintigraphy showed abnormal uptake in the spleen, a few small mediastinal lymph nodes, and subcutaneous tissue at the outside aspects of both thighs.

skin test was negative. A chest X-ray revealed no abnormalities such as BHL or reticular shadows (Fig. 1). An electrocardiogram revealed first-degree atrioventricular block, but not a complete atrioventricular block. Echocardiography did not reveal asymmetric septal hypertrophy. Laboratory findings were as follows: hemoglobin, $12.1 \mathrm{~g} / \mathrm{dL}$; white blood cell count, $5,100 / \mu \mathrm{L}$; platelet count, $15.3 \times 10^{4} / \mu \mathrm{L}$; total serum protein, $7.6 \mathrm{~g} / \mathrm{dL}$; serum albumin, $4.1 \mathrm{~g} / \mathrm{dL}$; urea nitrogen, $32 \mathrm{mg} / \mathrm{dL}$; serum creatinine, $7.24 \mathrm{mg} / \mathrm{dL}$; sodium, 136 $\mathrm{mEq} / \mathrm{L}$; potassium, $5.2 \mathrm{mEq} / \mathrm{L}$; chloride, $96 \mathrm{mEq} / \mathrm{L}$; calcium, $12.5 \mathrm{mg} / \mathrm{dL}$; phosphorus, $4.0 \mathrm{mg} / \mathrm{dL}$; C-reactive protein, $0.14 \mathrm{mg} / \mathrm{dL}$; immunoglobulin $\mathrm{G}$ ( $\mathrm{IgG}), 1,162.6 \mathrm{mg} / \mathrm{dL}$; immunoglobulin A (IgA), $391.6 \mathrm{mg} / \mathrm{dL}$; immunoglobulin $\mathrm{M}$ (IgM), $23.3 \mathrm{mg} / \mathrm{dL}$; iPTH, $9.0 \mathrm{pg} / \mathrm{mL}$; parathyroid hormone related protein $(\mathrm{PTHrP}), \quad<1.0 \mathrm{pmol} / \mathrm{L} ; \quad 1,25-$ dihydroxyvitamin $\mathrm{D} 3,55.0 \mathrm{pg} / \mathrm{mL}$; angiotensin-converting enzyme (ACE), $21.5 \mathrm{mU} / \mathrm{mL}$; lysozyme, $84.2 \mu \mathrm{g} / \mathrm{mL}$; and soluble interleukin-2 receptor, 3,737 U/mL. Bone scintigraphy with technetium-99 m methylene diphosphonate did not reveal any abnormal uptake. Radionuclide gallium scintigraphy revealed abnormal uptake in the spleen, a few small mediastinal lymph nodes, and subcutaneous tissue on the outside aspects of both thighs (Fig. 2). Ophthalmological screening revealed asymptomatic uveitis in only the left eye, although it had not been detected in May 2011. Based on the above findings, he was suspected to have sarcoidosis, but it seemed to be atypical. Therefore, malignant diseases producing 1,25-dihydroxyvitamin D3, such as malignant lymphoma, and other granulomatous diseases such as tuberculosis were considered as differential diagnoses. Although tissue diagnosis is needed for a definitive diagnosis, there 
were no typical pathological lesions available for biopsy. To establish a definitive diagnosis, we performed a skin biopsy from the outside aspect of his left thigh, where there was abnormal uptake of gallium without any skin manifestations of sarcoidosis. Noncaseating granulomas with multinucleated giant cells were revealed in the tissue biopsy (Fig. 3). Then we performed a bone-marrow biopsy for the assessment of malignant lymphoma, and no malignant finding was observed. The diagnosis of sarcoidosis was then ascertained. Steroid therapy was considered to treat the hypercalcemia. However, because sarcoidosis-related hypercalcemia generally has the potential to heal spontaneously, and because his hypercalcemia had slightly improved without any therapy after admission, he was discharged from the hospital in September 2011. The hypercalcemia then stabilized to an adjusted calcium level of 10.0 to $11.0 \mathrm{mg} / \mathrm{dL}$ for a while. However, his serum ACE and lysozyme levels were generally maintained at high levels, and his hypercalcemia flared again with an elevated serum 1,25-dihydroxyvitamin D3 level in January 2012. The left uveitis also worsened despite local steroid therapy, and uveitis subsequently occurred de novo in the right eye. In January 2012, oral prednisolone

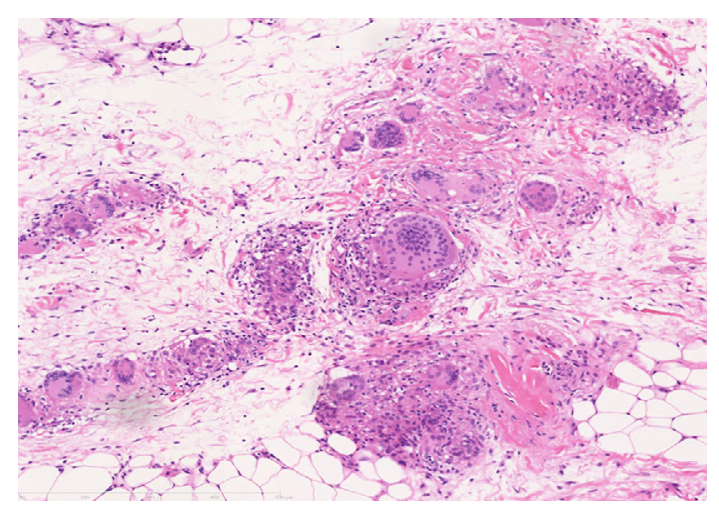

Figure 3. Skin biopsy from the outside aspect of the left thigh revealed noncaseating granulomas with multinucleated giant cells.
(PSL) was administered at $20 \mathrm{mg} /$ day. Within 1 month, the persistent hypercalcemia and bilateral uveitis improved rapidly, and the ACE, lysozyme, and 1,25-dihydroxyvitamin D3 levels simultaneously decreased. The PSL dose was gradually decreased by $5 \mathrm{mg}$ every 4 weeks, then continued at 5 $\mathrm{mg} /$ day as a maintenance dose for 1 year. The sarcoidosis has not flared up throughout the follow-up (Fig. 4).

\section{Discussion}

The present case of sarcoidosis in a chronic dialysis patient was diagnosed in the wake of sarcoidosis-related hypercalcemia without the presence of any common systemic clinical manifestations. This case was very difficult to make a diagnosis because of many atypical features as sarcoidosis, lack of common symptoms and elderly onset and so on. Therefore we considered differential diagnoses, especially tuberculosis and malignant lymphoma producing 1,25dihydroxyvitamin D3. But tuberculosis was interpreted as negative by reasons of negative tuberculin skin test and good response to steroid therapy, and malignant lymphoma was also negative for the sake of pathological findings.

To the best of our knowledge, only 23 cases of sarcoidosis in chronic dialysis patients have been reported (5-24). Their clinical characteristics, laboratory data, and treatment are listed in Table 2. Nineteen of all 23 cases had sarcoidosis-related hypercalcemia with an adjusted serum calcium level of $>10.5 \mathrm{mg} / \mathrm{dL}$ at diagnosis. Ten of these patients developed sarcoidosis-related hypercalcemia of less than the upper limit of the normal level of serum 1,25dihydroxyvitamin D3 (60 pg/mL). Eleven patients lacked common systemic clinical manifestations, BHL, uveitis, or EN at diagnosis. Only six patients had BHL, six had uveitis, and two had EN at diagnosis. Nine patients had atypical organ involvement, arthrosis, or muscle, nerve, or bone marrow involvement. Sixteen patients underwent steroid therapy, seven of whom responded well to PSL at $<30 \mathrm{mg} /$ day, although a dose of 30 to $40 \mathrm{mg} /$ day is generally recom-

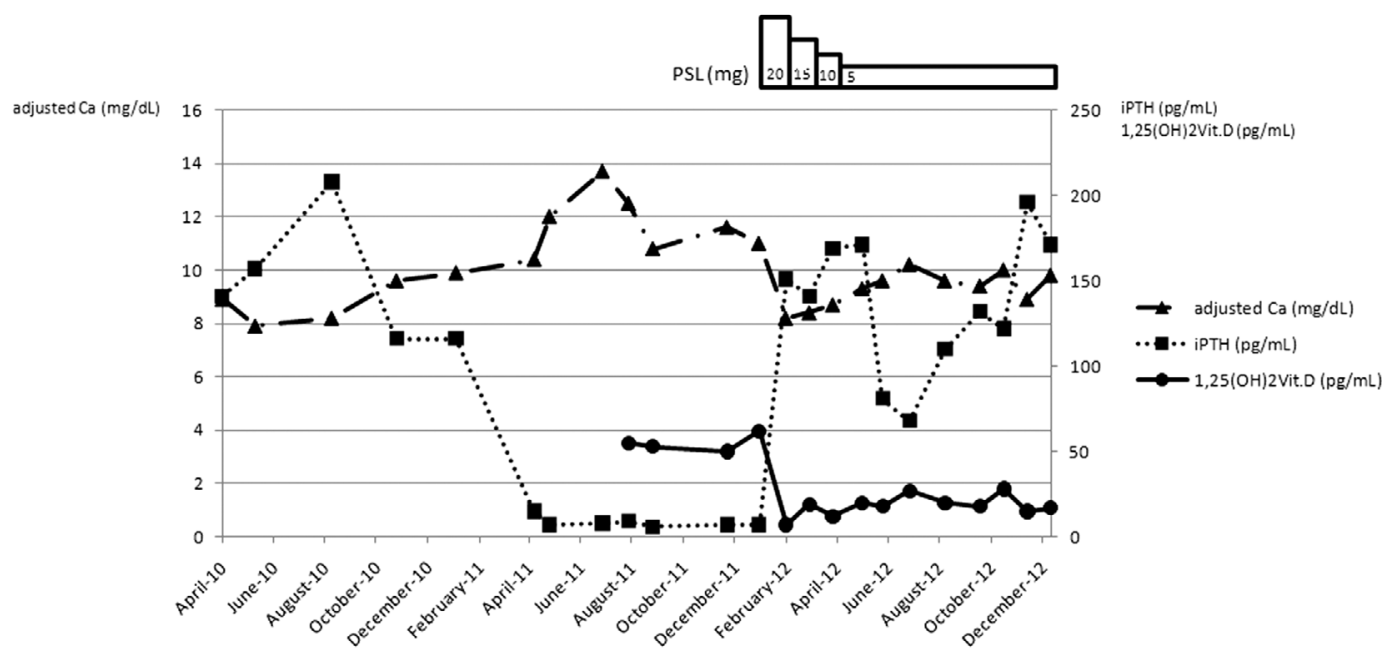

Figure 4. Clinical course. 
Table 2. Clinical Characteristics, Laboratory Data, and Treatment of Cases of Sarcoidosis in Chronic Dialysis Patients

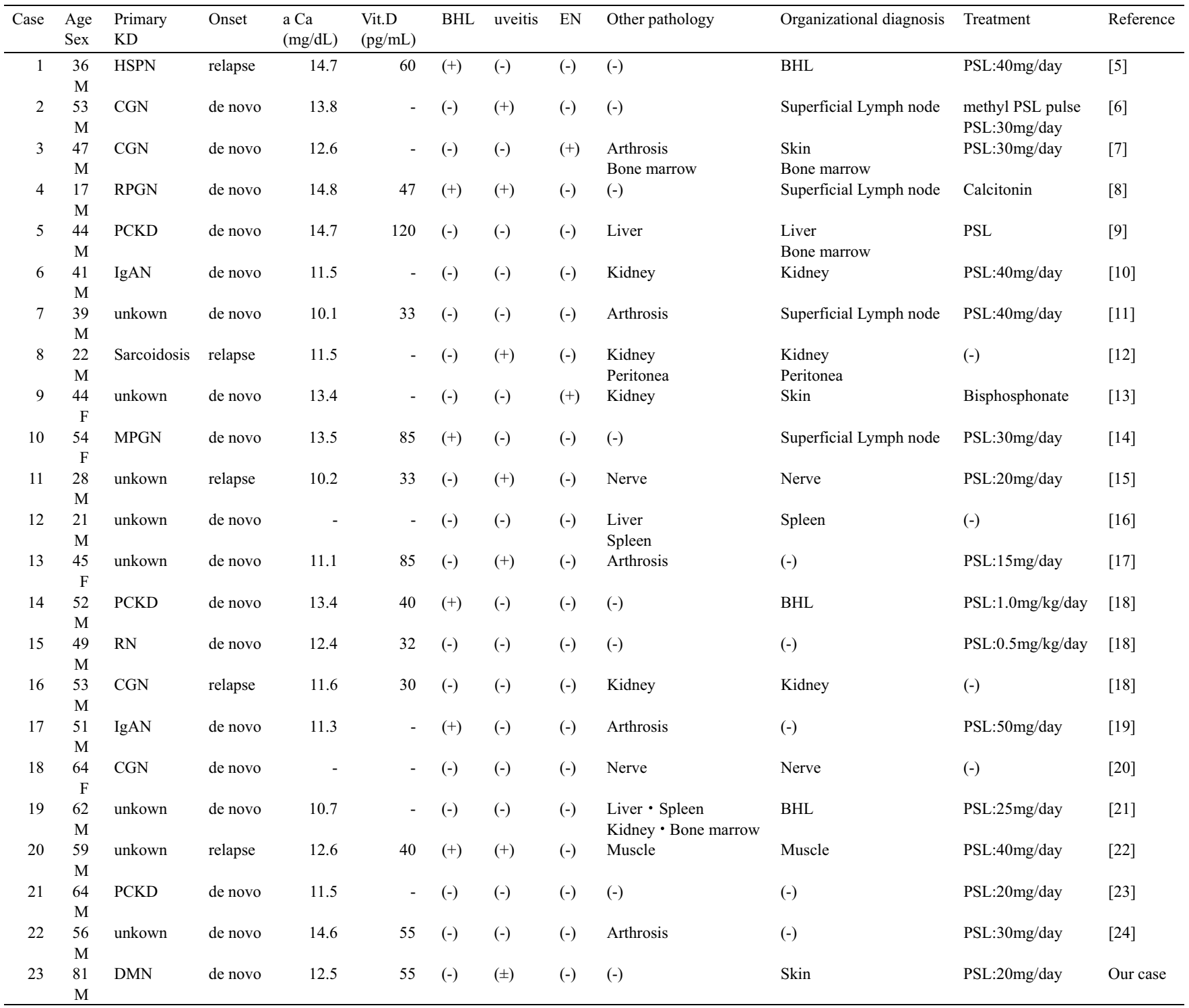

Abbreviations: primary KD: primary kidney disease, a Ca: adjusted Calcium level, Vit.D: 1,25-dihydroxyvitamin D3 level, BHL: bihilar lymphadenopathy, EN: erythema nodosum, HSPN: Henoch-Schönlein purpura nephritis, CGN: chronic glomerulonephritis, RPGN: rapidly progressive glomerulonephritis, PCKD: polycystic kidney disease, IgAN: immunoglobulin A nephropathy, MPGN: membranoproliferative glomerulonephritis, RN: reflux nephropathy, DMN: diabetic nephropathy, PSL: prednisolone, M: male, F: female

mended for sarcoidosis-related hypercalcemia (25). To review these reports, sarcoidosis in chronic dialysis patients is considered to be a rare condition. A diagnosis of sarcoidosis is often provided in the wake of sarcoidosis-related hypercalcemia without the presence of most common clinical manifestations. In such cases, sarcoidosis-related hypercalcemia sometimes improves with relatively low doses of PSL. In patients with comorbid organ involvement, atypical organ involvement frequently occurs. Notably, however, this review is based on only a few case reports and contains publication bias.

We suppose that these clinical features may arise from an unique physiology of 1,25-dihydroxyvitamin D3, a main cause of sarcoidosis-related hypercalcemia, and a specific immune deficiency in chronic dialysis patients.

The mechanism of sarcoidosis-related hypercalcemia is considered to involve 1,25-dihydroxyvitamin D3 produced by macrophages in noncaseating granulomas (26). In general, 1,25-dihydroxyvitamin D3 is produced in the kidneys, but this extrarenal production of 1,25-dihydroxyvitamin D3 plays a central role in the development of hypercalcemia in patients with sarcoidosis. Various cytokines, especially interferon- $\gamma$, released spontaneously from macrophages in noncaseating granulomas, are known to induce $1 \alpha$ hydroxylase, which catalyzes the conversion of 25hydroxyvitamin D3 to 1,25-dihydroxyvitamin D3 (27). Hyperproduction of 1,25-dihydroxyvitamin D3 triggers hypercalcemia because of increased bone resorption and calcium absorption from the gastrointestinal tract, leading to suppression of iPTH. On the other hand, steroid therapy for hypercalcemia is considered to suppress the induction of $1 \alpha$ hydroxylase in macrophages and lead to normalization of 1,25-dihydroxyvitamin D 3 (28). Therefore 1,25dihydroxyvitamin D3 is associated with the activity of sar- 
coidosis (29). Indeed, in the present case, the serum concentrations of calcium and iPTH were well linked to the variation in the serum 1,25-dihydroxyvitamin D3 level (Fig. 2). However, it should be noted that the serum 1,25dihydroxyvitamin D3 level at the onset of sarcoidosis-related hypercalcemia was within the normal range $(20-60 \mathrm{pg} / \mathrm{mL})$. In patients with chronic renal failure, especially during chronic dialysis, the serum 1,25-dihydroxyvitamin D3 level reportedly tends to decrease in proportion to the residual renal function (30). Under its influence, chronic dialysis patients become more sensitive to 1,25-dihydroxyvitamin D3 than nondialysis patients. Therefore, they often experience hypercalcemia even within the normal range of 1,25dihydroxyvitamin D3. Thus, we consider that hypercalcemia is strongly associated with sarcoidosis in chronic dialysis patients. We also consider that sarcoidosis-related hypercalcemia appears early in the course of sarcoidosis in chronic dialysis patients. Actually, in the present case, there were no common systemic clinical manifestations without hypercalcemia at diagnosis. Bilateral uveitis, one of the typical findings of sarcoidosis, subsequently developed in a delayed manner.

Then chronic dialysis patients have an impaired immune response that accounts for their susceptibility to infections (31). The immunodeficiency of chronic dialysis patients results from a disturbance in the function of various cell types involved in immune defenses, including polymorphonuclear leukocytes, monocytes, and T lymphocytes (32). In such patients, the immunodeficiency of $\mathrm{T}$ lymphocytes, especially T-helper lymphocytes 1 (Th1), is important. Although the cause of sarcoidosis is incompletely understood, the pathogenesis of sarcoidosis is considered to involve antigen exposure in a genetically susceptible host, resulting in typical granulomatous inflammation with a prominent Th1cell-mediated immune response (1). Therefore, Th1 immunodeficiency in chronic dialysis patients may result in hypoplasia of granulomas. It may also decrease the risk of developing sarcoidosis. Furthermore, because causative antigens are not enclosed by a typical granuloma, they may spread hematogenously throughout the body, potentially leading to atypical organ involvement. We suppose that the few reports of sarcoidosis in chronic dialysis patients may be because it is often difficult to achieve a definitive diagnosis by biopsy. The present case had an absence of typical findings of sarcoidosis. Fortunately, however, a skin biopsy taken from an area of abnormal gallium uptake despite no macroscopic skin abnormalities revealed noncaseating granulomas with multinucleated giant cells. The diagnosis of sarcoidosis was thus ascertained. In such a sense, gallium scintigraphy is often helpful in evaluating the progression of sarcoidosis (33).

In conclusion, the present report describes a case of sarcoidosis in a chronic dialysis patient diagnosed in the wake of sarcoidosis-related hypercalcemia without the presence of any common systemic clinical manifestations. A review of sarcoidosis in chronic dialysis patients showed that a charac- teristic of sarcoidosis in chronic dialysis patients is that the diagnosis of sarcoidosis is often achieved in the wake of sarcoidosis-related hypercalcemia without the presence of most common clinical manifestations. This characteristic may arise from the specific immune deficiency and the unique physiology of 1,25-dihydroxyvitamin D3, a main cause of sarcoidosis-related hypercalcemia, in chronic dialysis patients. These clinical features may be useful to understand the pathogenesis of sarcoidosis. Despite the fact that sarcoidosis is a rare condition in chronic dialysis patients, and even when no typical findings of sarcoidosis are present, one should still consider sarcoidosis as a differential diagnosis.

The authors state that they have no Conflict of Interest (COI).

\section{References}

1. O'Regan A, Berman JS. Sarcoidosis. Ann Intern Med 156: ITC5$1,2012$.

2. Winterbauer RH, Belic N, Moores KD. Clinical interpretation of bilateral hilar adenopathy. Ann Intern Med 78: 65-71, 1973.

3. Rothova A, Bultenhuis HJ, Meenken C. Uveitis and systemic disease. Br J Ophthalmol 76: 137-141, 1992.

4. Baughman RP, Teirstein AS, Judson MA. Case Control Etiologic Study of Sarcoidosis (ACCESS) research group. Clinical characteristics of patients in a case control study of sarcoidosis. Am J Respir Crit Care Med 164: 1885-1889, 2001.

5. Barbour GL, Coburn JW, Slatopolsky E, Norman AW, Horst RL. Hypercalcemia in an anephric patient with sarcoidosis: evidence for external generation of 1,25-dihydroxyvitamin D. N Engl J Med 305: 440-443, 1981.

6. Ebara H, Kanematsu M, Deguchi T, Kuriyama M, Ban Y, Kawada Y. Sarcoidosis with hypercalcemia and disturbance of consciousness in a hemodialysis patient. Nippon Toseki igakkai Zasshi 24: 1505-1508, 1991.

7. Watanabe T, Kudo K, Hukuda K, et al. Polyarthritis due to sarcoidosis in continuous ambulatory peritoneal dialysis patient. Yamagata Saiseikan Medical Journal 16: 115-123, 1991.

8. Tamae S, Kawamura K, Kamiyama Y, et al. A case of patient with sarcoidosis with end-stage renal disease undergoing hemodialysis. Jin to Toseki 33: 169-172, 1992.

9. Kalantar-Zadeh K, Neumayer HH, Wunsch PH, Luft FC. Hypercalcemia and sarcoidosis in an anephric dialysis patient. Nephrol Dial Transplant 9: 829-831, 1994.

10. Hrrero JC, Morales E, Dominguez-Gil B, et al. Reactivation of multisystemic sarcoidosis after immunosuppression withdrawal in a transplanted patient returning to chronic dialysis. Nephrol Dial Transplant 13: 3280-3281, 1998.

11. Naito T. Progressive tumoral calcinosis as the presenting feature of sarcoidosis in a patient on haemodialysis treatment. Nephrol Dial Transplant 14: 2716-2719, 1999.

12. Takada D, Kashiwagi T, Kaneko T, et al. Continuous Ambulatory Peritoneal Dialysis (CAPD) patient with peritoneal sarcoidosis leading of dialysis efficiency. Nihon Naika Gakkai Zasshi 90: 1342-1344, 2001.

13. Barnard S, Marshall R, Parry RG, Johnston P. Sarcoid presenting with hypercalcemia and erythema nodosum in a haemodialysis patient. Nephrol Dial Transplant 17: 175, 2002.

14. Kuwae N, Oshiro Y, Nakazato S. Sarcoidosis and hypercalcemia in a patient undergoing hemodialysis. Nippon Jinzo Gakkai Shi 45: 98-103, 2003.

15. Tanaka K, Tanimoto H, Sakai K, et al. Recurrent of sarcoidosis in 
a hemodialysis patient confirmed by abnormal calcium metabolism. Clin Calcium 15: 83-85, 2005.

16. Nishioka H, Hirabayashi K, Hazano K, Yamaguchi T, Toguchi $\mathrm{K}$, Nakabayashi H. A case of successful surgery for splenic sarcoidosis with giant splenomegaly and hypersplenism. Nippon Rinsho Geka Gakkai Zasshi 66: 1739-1742, 2005.

17. Sato T, Tsuru T, Hagiwara K, et al. Sarcoidosis with acute recurrent polyarthritis and hypercalcemia. Intern Med 45: 363-368, 2006.

18. Huart A, Kamar N, Lanau JM, Dahmani A, Durand D, Rostaing L. Sarcoidosis-related hypercalcemia in 3 chronic hemodialysis patients. Clin Nephrol 65: 449-452, 2006.

19. Quack I, Woznowski M, Schieren G, et al. Hypercalcemia after transplant nephrectomy in a hemodialysis patient: a case report. J Med Case Reports 3: 164, 2007.

20. Tanaka K, Tsuchiya K, Yamaoka K, Sasaki T. A case of solitary sarcoid nodule at lumbar root. Orthopedics \& Traumatology 56 601-603, 2007.

21. Isobe Z, Suga T, Aoki Y, et al. A case of sarcoidosis with hypoxia showing slight ground glass opacities on chest CT. Nihon Kokyuki Gakkai Zasshi 46: 899-903, 2008.

22. Yamada S, Taniguchi M, Tsuruya K, Iida M. Recurrent sarcoidosis with psoas muscle granuloma and hypercalcemia in a patient on chronic haemodialysis. Nephrology 14: 452-453, 2009.

23. Ochi A, Wakai S, Nakayama $\mathrm{K}$, et al. A case of sarcoidosis followed by hypercalcemia in a hemodialysis patient. Nippon Toseki igakkai Zasshi 44: 649-654, 2011.

24. Horikoshi R. Tumoral calcinosis associated with hypercalcemia in a patient with chronic renal failure. Clin Exp Nephrol 15: 154158, 2011.

25. Newman LS, Rose CS, Maier LA. Sarcoidosis. N Engl J Med
336: 1224-1234, 1997.

26. Sharma OP. Vitamin D, calcium, and sarcoidosis. Chest 109: 535539, 1996.

27. Koeffler HP, Reichel H, Bishop JE, Norman AW. Gannmainterferon stimulates production of 1,25-dihydroxyvitamin D3 by normal human macrophages. Biochem Biophys Res Commun 127: 596-603, 1985.

28. Robinson BW, Mclemore TL, Crystal RG. Gamma interferon is spontaneously released by alveolar macrophages and lung $\mathrm{T}$ lymphocytes in patients with pulmonary sarcoidosis. J Clin Invest $\mathbf{7 5}$ : 1488-1495, 1985.

29. Inui N, Murayama A, Sasaki S, et al. Correlation between 25hydrpxyvitamin D3 $1 \alpha$-hydroxylase gene expression in alveolar macrophages and the activity of sarcoidosis. Am J Med 110: 687693, 2001.

30. Slatopolsky E, Gray R, Adams MD, et al. The pathogenesis of secondary hyperparathyroidism in early renal failure. In: Vitamin D: basic research and its clinical application (proceedings from the Fourth International Workshop on Vitamin D. Berlin, West Germany). defences and immunologic alterations associated with chronic hemodialysis. Ann Intern Med. 93. Norman AW, Schaefer K, Herrath DV, et al, Eds. 1980: 597-613.

31. Goldblum SE, Reed WP. Host defences and immunologic alterations associated with chronic hemodialysis. Ann Intern Med 93: 597-613, 1980.

32. Kelly CJ. T cell function in chronic renal failure and dialysis. Blood Purif 12: 36-41, 1994.

33. Johnson DG, Johnson SM, Harris CC, Piantadosi CA, Blinder RA, Coleman RE. Ga-67 uptake in the lung sarcoidosis. Radiology 150: 551-555, 1984.

(C) 2013 The Japanese Society of Internal Medicine http://www.naika.or.jp/imonline/index.html 